\title{
Novel method for the generation of stable radiation from free-electron lasers at high repetition rates
}

\author{
Sven Ackermann $\odot,{ }^{*}$ Bart Faatz, ${ }^{\dagger}$ Vanessa Grattoni, Mehdi Mohammad Kazemi, Tino Lang, \\ Christoph Lechner, Georgia Paraskaki, ${ }^{\ddagger}$ and Johann Zemella \\ Deutsches Elektronen-Synchrotron DESY, 22607 Hamburg, Germany \\ Gianluca Geloni, Svitozar Serkez, and Takanori Tanikawa \\ European XFEL, 22869 Schenefeld, Germany \\ Wolfgang Hillert $\odot$ \\ University of Hamburg, 22761 Hamburg, Germany
}

(Received 17 April 2020; accepted 17 July 2020; published 29 July 2020)

\begin{abstract}
For more than a decade free-electron lasers (FELs) have been in operation, providing scientists from many disciplines with the benefits of ultrashort, nearly transversely coherent radiation pulses with wavelengths down to the Ångstrom range. If no further techniques are applied, the FEL will only amplify radiation from the stochastic distributed electron density in the electron bunch. Contemporary developments aim at producing stable and single-mode radiation by preparing an electron bunch with favorable longitudinal electron density distributions using magnets and conventional laser pulses (seed), hence the name "seeding." In recent years, short wavelength FELs at high electron beam energies and high repetition rates were proposed and built. At those repetition rates, an external seed with sufficient power to manipulate the electron beam cannot be provided by present state-of-the-art laser systems, thus no external seeding scheme could be applied yet. In this paper, we present ways to seed FELs to generate short wavelength radiation at high repetition rates, making use of tested electron beam manipulation schemes. For our parameter study, we used the parameters of FLASH, the free-electron laser in Hamburg. First simulations are presented, showing the feasibility of the method proposed.
\end{abstract}

DOI: 10.1103/PhysRevAccelBeams.23.071302

\section{INTRODUCTION}

In most free-electron lasers (FELs), the radiation generation process referred to as self-amplified spontaneous emission (SASE) radiation is initiated only by the stochastic noise in the electron density distribution in the bunch, called shot noise. Therefore, the amplified radiation suffers from low longitudinal coherence and large shot-to-shot fluctuations. Several techniques, consolidating under the term "seeding," have been conceived to provide favorable electron beam density distributions as initial conditions to overcome this limitation.

\footnotetext{
*sven.ackermann@desy.de

Present address: Shanghai Advanced Research Institute, Shanghai, China.

georgia.paraskaki@desy.de

Published by the American Physical Society under the terms of the Creative Commons Attribution 4.0 International license. Further distribution of this work must maintain attribution to the author(s) and the published article's title, journal citation, and DOI.
}

The linear accelerators (linacs) providing the electron beam for the FEL can be divided into two categories, depending on the used technology: normal conducting (warm), operating at repetition rates in the order of $100 \mathrm{~Hz}$, and superconducting (cold) at repetition rates up to several MHz. At the moment, only two such cold machines exist: FLASH at DESY [1,2], and the European XFEL (EuXFEL) [3]. They operate in what is called burst-mode operation, which means that they produce short bursts of electron bunches during several hundreds of microseconds at a repetition rate of $10 \mathrm{~Hz}$, resulting in several thousand to several ten thousand electron bunches per second. Future machines, based on the same technology, will be running in continuous mode (cw), thus producing millions of bunches per second with equidistant separation. LCLS-II [4] and SHINE [5] are under construction. Seed lasers at repetition rates of these superconducting accelerators with sufficient pulse energies are currently beyond state-of-the-art technology for all of them.

In seeding schemes that use external lasers, only a fraction of the laser power is actually coupled to the electron beam. Therefore, instead of increasing the repetition rate of the seed laser, we propose to recirculate the laser 
to seed the next bunch in a bunch train. A variation of this idea has been studied already 25 years ago [6-10], to cite only a few. All studies mentioned above have in common that the application of seeding techniques was not investigated.

Another idea is to use the spent electron beam just before it is dumped to generate radiation which is stored in an oscillator. This radiation is used to seed the main FEL. This method is called "resonator first scheme" [11]. This requires additional beam line elements and increases the geometric footprint of the FEL. All oscillator-based approaches have in common that the radiation has to build up over several bunches, and when driven to saturation, the energy spread can become large which is disadvantageous for the needed quality of energy modulation. Using an external seed laser pulse provides precise control over the seeding start-up conditions. In addition, choosing the right modulator length and initial seed laser power for a given wavelength can keep the induced energy spread in the modulator controlled, and far from saturation. This is verified with simulations, shown in Sec. IVA, and in addition, a detailed study is underway.

With the development in mirrors, simulation codes, improvement of the beam quality from the injectors, and increased knowledge of FEL schemes that are possible, we have worked out several possibilities that will be described in this paper. The combination of an optical, resonator-like seed recirculation feedback system (SRS) with high-gain harmonic generation (HGHG) [12-16], one method to reach shorter wavelengths, is briefly introduced in Sec. II. Further methods to reach shorter wavelengths, which have been developed in the period since then [17], namely cascaded high-gain harmonic generation (cascaded HGHG) [18-20], and Echo-Enabled Harmonic Generation (EEHG) [21-23] are discussed in Sec. III. For the HGHG case, simulations have been performed, exploring the feasibility of the concept. They are presented in Sec. IV. Further studies are underway to explore the limits of the scheme both on technical and a physics level. The final Sec. V will summarize the work and give an outlook onto further studies to come.

\section{ACHIEVING HIGH REPETITION RATES}

One method to reach shorter wavelengths is high-gain harmonic generation seeding, as depicted in Fig. 1. It needs an undulator, called modulator whose resonant wavelength $\lambda_{\text {Seed }}$ is tuned in a way such that it fits the seed laser wavelength at the given electron beam energy [12-16]. Thus, within the modulator an energy exchange between seed and the initial electron beam, as shown in Fig. 2(a), will take place, resulting in an energy modulation in the electron beam [Fig. 2(b)]. Downstream of the modulator, the dispersive strength of a bunching chicane is used to convert the energy modulation of the electron beam into an electron density modulation rich in harmonic content [See Fig. 2(c) and (d)]. A final long undulator, called radiator, is

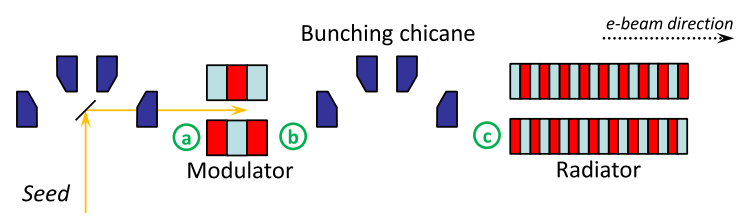

FIG. 1. Layout for the HGHG setup. The electron beam is energy-modulated by an external laser at long wavelength $\lambda_{\text {Seed }}$ in the modulator. After converting the energy modulation into a density modulation in the bunching chicane, the electron beam radiates at a harmonic wavelength $\lambda_{n}=\lambda_{\text {Seed }} / n$ of the imprinted wavelength in the radiator, which is typically tuned between $n=5$ and $n=12$.
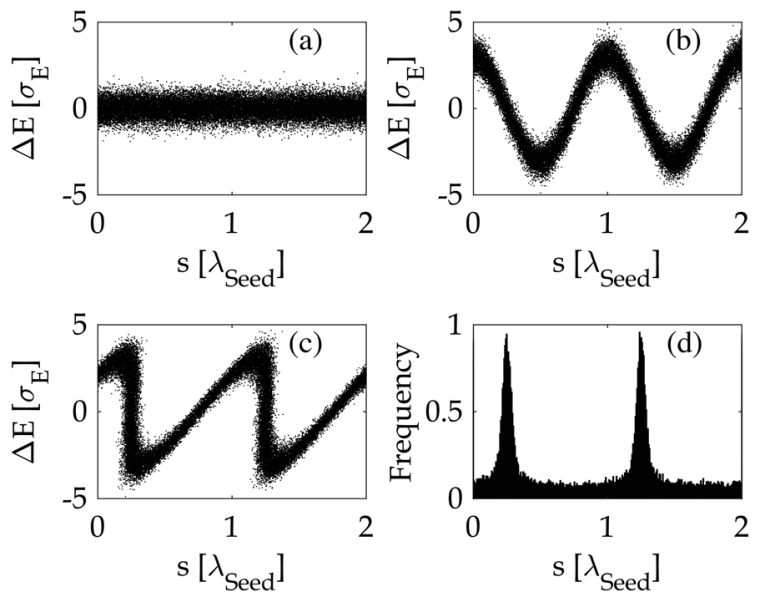

FIG. 2. Evolution of the longitudinal phase space distribution during the HGHG process. The horizontal axis is the bunch coordinate $s$ in units of seeding wavelength $\lambda_{\text {Seed }}$, while the vertical axis shows the deviation from the central energy $\Delta \mathrm{E}$ in units of the uncorrelated energy spread $\sigma_{\mathrm{E}}$. Shown is the energy spread at 3 locations $a, b$, and $c$ as indicated in Fig. 1 and a histogram of the electron density at location $c$ in (d). One can see that a larger fraction of the electrons is confined within distances smaller than the seed wavelength, thus having a higher harmonic content.

tuned to a harmonic $\lambda_{n}=\lambda_{\text {Seed }} / n$ of the seed laser. Therefore, the properly manipulated electron bunch with an electron beam energy $E_{0}$ will radiate at this wavelength. Typical laser systems used in external seeding today use wavelengths in the UV range. The maximum harmonic is limited by the electron beam energy spread: The laserinduced energy modulation $\Delta \mathrm{E}$ needs to be about as large as the energy spread of the electron bunch $\sigma_{\mathrm{E}}$ multiplied by the harmonic number $n: \Delta \mathrm{E} \approx n \sigma_{\mathrm{E}}$. For reasonable FEL performance the relative uncorrelated energy spread must not exceed the FEL parameter $\rho: \sigma_{\mathrm{E}} / \mathrm{E}_{0} \lesssim \rho$. This leads to $n<\rho \mathrm{E}_{0} / \sigma_{\mathrm{E}}$, and practically limits the number of harmonics to $n \leq 15$, which has been shown in [14]. With the seed laser wavelength $\lambda_{\text {Seed }}$ limited to between $200 \mathrm{~nm}$ and $300 \mathrm{~nm}$, the FEL wavelength cannot become shorter than around $14 \mathrm{~nm}$. 


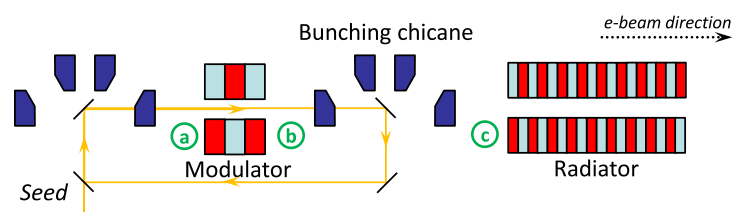

FIG. 3. Layout for the HGHG setup with an optical, resonatorlike seed recirculation feedback system. The underlying principle is the same as with HGHG shown in Fig. 1. However, a seed laser pulse is only needed once per macropulse and the optical, resonator-like seed recirculation feedback system is used to maintain the seed power for successive electron bunches in the burst.

Laser systems with the characteristics required for the implementation of the HGHG scheme are available in the kHz-range. As the repetition rate of the accelerator increases, the average power of the laser needs to increase as well. Although development continuously improves the laser systems, the repetition rate is at the moment limited to between $100 \mathrm{kHz}$ and $1 \mathrm{MHz}$ with insufficient pulse energies. Although this covers a significant number of FEL facilities, there is a trend toward shorter wavelengths and high repetition rates. With FLASH, EuXFEL, LCLS-II, and SHINE, the interest in reaching seeding at higher repetition rates is growing and it can be expected that this will become more in the coming decades. Therefore, we have been looking at alternative approaches.

At the end of the modulator a radiation pulse is emitted which consists partly of the initial one, superimposed with the radiation generated during the passage of the electron bunch. Instead of increasing the repetition rate of the seed laser, we recirculate this radiation leaving the modulator to seed the next bunch in the bunch train. However, due to the interaction with the electron bunch, this seed pulse needs to be mode-matched and focused before it can be used to seed again. The layout of such SRS FEL facility is shown in Fig. 3. As in all optical resonators, the pulse power is not conserved. Therefore, the modulator also needs to amplify the recirculating pulse. Consequently, the SRS around the modulator has several functions: (i) Transport the radiation after interaction with the electron beam back to the modulator entrance to interact with the next, fresh electron bunch. (ii) Refocus the photon beam to obtain the correct size at the modulator entrance, and focal point. (iii) Attenuate the pulse energy to the correct seed power.

As will be discussed later, the SRS, which is in Fig. 3 simplified as a ring resonator, can meet all the required properties needed.

\section{ACHIEVING SHORT WAVELENGTHS AT HIGH REPETITION RATES}

The modified HGHG scheme described in the section above allows operation at the high repetition rates of superconducting linacs. However, it is still limited to an harmonic number $n \leq 15$. Three options to generate shorter wavelengths, all of which have been demonstrated, are discussed below.

\section{A. Use of a high-harmonic generation source}

Instead of using an optical laser to seed the FEL, one can also use a high-harmonic generation (HHG) source. The efficiency of such sources results in micro-joules of pulse energy at low repetition rates down to a wavelength of around $50 \mathrm{~nm}$, and allows extending the wavelength range to $4 \mathrm{~nm}$ or $5 \mathrm{~nm}$. Therefore, combining a conventional seed laser wavelength with a tunable HHG source between $50 \mathrm{~nm}$ and $100 \mathrm{~nm}$, the complete wavelength range from $4.2 \mathrm{~nm}$ to $100 \mathrm{~nm}$ is accessible.

However, HHG sources are less stable than conventional lasers. Therefore, we look at more advanced, but still practically demonstrated, seeding schemes that can be combined with an SRS. Nevertheless, the use of HHG sources is assumed for the simulations presented in Sec. IV.

\section{B. Cascaded high-gain harmonic generation}

In order to overcome the short-wavelength limitations of the HGHG scheme that we discussed above, a subsequent HGHG stage can be realized, hence the name "cascaded HGHG" [18-20]. The setup for this method is depicted in Fig. 4. The radiation from the first radiator is used as a seed pulse for the second modulator and needs to hit an unspoiled part of the electron beam, which is achieved by the use of a chicane called delay line. The final radiation of the cascaded HGHG is then the product of the harmonic generated in each of the two HGHG steps.

Because the 2nd stage of the cascaded HGHG uses the FEL radiation of the first stage, only the first stage needs an SRS to allow for high repetition rates. Therefore, this scheme can reach shorter wavelengths while the SRS can work at relatively long wavelength of $200 \mathrm{~nm}$ to $300 \mathrm{~nm}$.

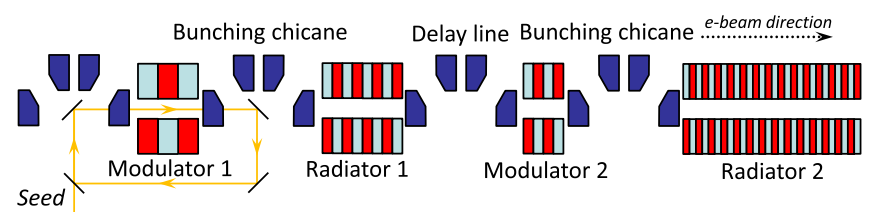

FIG. 4. Layout for the cascaded HGHG setup. The FEL pulse from the 1st HGHG stage on the left is used on an unbunched part of the electron bunch in a second stage. Therefore, the seed pulse needs to be significantly shorter than the electron bunch and a delay line is used to shift the electron bunch with respect to the FEL pulse, applying the so-called fresh-bunch technique. Because both HGHG stages use an up-conversion of the seed wavelength, much shorter wavelength can be reached, typically a factor of 50 or more compared to the original seed. 


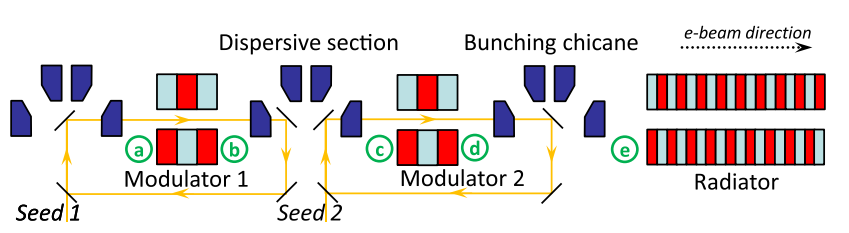

FIG. 5. Layout for the EEHG setup. Here, only one radiator is needed.

\section{Echo-enabled harmonic generation}

The EEHG scheme [21-23] uses the chicane behind the first modulator to overshear the electron beam over many wavelengths of the seed laser after it has been modulated. Therefore, this chicane needs to have a large dispersive strength. The second modulator and second bunching chicane manipulates the beam as in a normal HGHG scheme, leading to regions of higher bunching at smaller wavelengths. An example for the evolution of the longitudinal phase-space distribution is shown in Fig. 6, while the basic layout can be found in Fig. 5. In this case
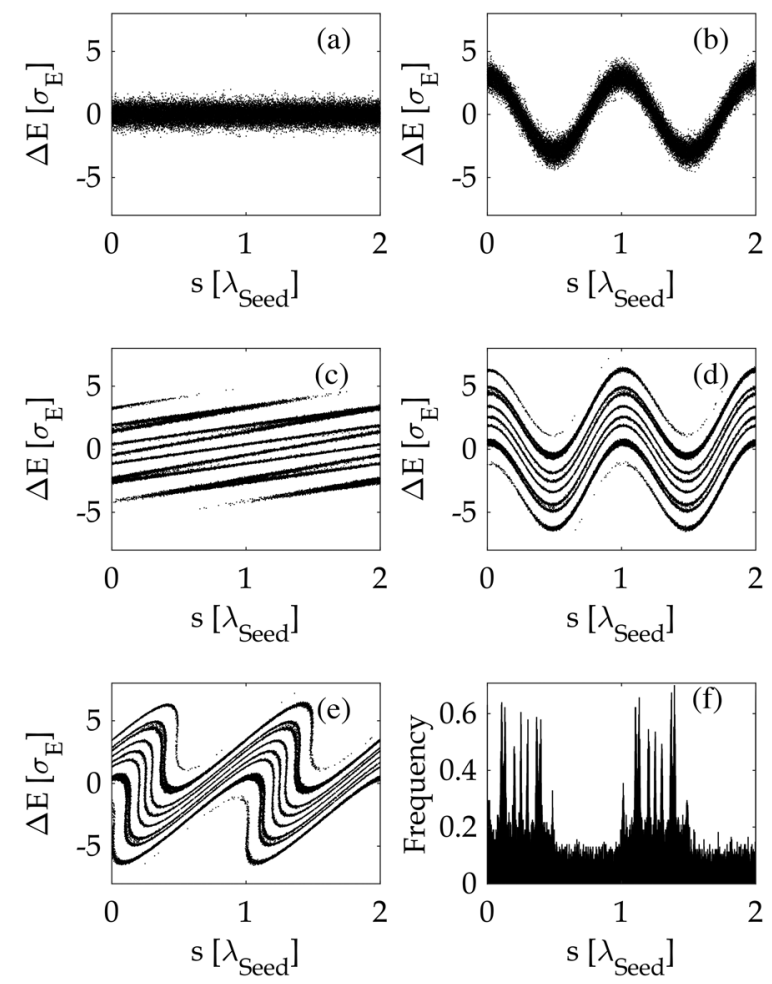

FIG. 6. Evolution of the longitudinal phase space distributions during the EEHG process. The horizontal axis is the bunch coordinate $s$ in units of seeding wavelength $\lambda_{\text {Seed }}$, while the vertical axis shows the deviation from the central energy $\Delta \mathrm{E}$ in units of the uncorrelated energy spread $\sigma_{\mathrm{E}}$. Shown is the energy spread at 5 locations $a, b, c, d$ and $e$ as indicated in Fig. 5 and a histogram of the electron density at location $e$ in (f). One can see that a larger fraction of the electrons is confined within distances much smaller than the seed wavelength, thus having a much higher harmonic content than in Fig. 2(d). bunching at harmonics higher than the 100th can be achieved [23].

Because two seeds are needed in the EEHG scheme, two SRS are included in Fig. 5. However, in most cases, both SRS s will be at moderate wavelengths.

\section{FIRST MULTIPASS HGHG SIMULATIONS}

In this section, we show simulation results of a highrepetition rate HGHG FEL which includes an optical feedback system, as described above and shown in Fig. 3. We refer to this scheme as a multipass HGHG, while the conventional HGHG scheme, which does not include an optical feedback system, is referred to as a single-pass HGHG. For our simulations, we use parameters that are expected for the FEL FLASH in Hamburg after the planned upgrades (See Table I).

FLASH is the first FEL user facility in the soft X-ray regime and has been in operation since 2005 [1,2]. It is also the only Soft X-ray / XUV-FEL based on superconducting technology, which produces up to 5000 bunches per second in 10 bursts up to $500 \mu \mathrm{s}$. Since 2016, FLASH runs two FELs simultaneously from the same accelerator $[24,25]$. In the future, FLASH will be upgraded to become the first seeded FEL driven by superconducting acceleration modules, and having a beam energy of $1.35 \mathrm{GeV}$ [26].

The target wavelengths we want to cover exploiting this scheme, using the two operating points in beam energy foreseen for FLASH in the future, are (i) $20 \mathrm{~nm}$ to $60 \mathrm{~nm}$ at an electron beam energy of $750 \mathrm{MeV}$ (ii) $4 \mathrm{~nm}$ to $20 \mathrm{~nm}$ at an electron beam energy of $1350 \mathrm{MeV}$.

Based on these operating points, this simulation study has been performed with seed lasers of $300 \mathrm{~nm}, 200 \mathrm{~nm}$, $100 \mathrm{~nm}$ and $50 \mathrm{~nm}$ and up to the 12th harmonic of each wavelength, therefore in a range between $4.17 \mathrm{~nm}$ and $60 \mathrm{~nm}$. We have selected to show the results of the 12th harmonic of a $50 \mathrm{~nm}$ seed laser which is the most challenging case and in addition, the 5th harmonic of

TABLE I. Simulation parameters for the conducted simulations.

\begin{tabular}{lcc}
\hline \hline & \multicolumn{2}{c}{ Electron beam } \\
\hline Energy & $750 \mathrm{MeV}$ & $1350 \mathrm{MeV}$ \\
Uncorrelated energy spread & $120 \mathrm{keV}$ & $120 \mathrm{keV}$ \\
Peak current & $1 \mathrm{kA}$ & $1 \mathrm{kA}$ \\
Normalized emittance & $1 \mathrm{~mm} \mathrm{mrad}$ & $1 \mathrm{~mm} \mathrm{mrad}$ \\
& \multicolumn{2}{c}{ Seed laser } \\
Duration (FWHM) & $78 \mathrm{fs}$ & $78 \mathrm{fs}$ \\
Wavelength & $300 \mathrm{~nm}$ & $50 \mathrm{~nm}$ \\
Input peak power & $1 \mathrm{MW}$ & $68.9 \mathrm{MW}$ \\
& Undulator parameters \\
$K_{\text {rms }}$ modulator & 4.53 & 3.26 \\
$K_{\text {rms }}$ radiator & 2.76 & 0.97 \\
$\lambda_{u}$ modulator & $60 \mathrm{~mm}$ & $60 \mathrm{~mm}$ \\
$\lambda_{u}$ radiator & $30 \mathrm{~mm}$ & $30 \mathrm{~mm}$ \\
\hline \hline
\end{tabular}


the $300 \mathrm{~nm}$ seed laser as an example of a longer wavelength for completeness.

Multi-pass HGHG follows the same principles as a single-pass HGHG introduced in Sec. II. The difference is that we use the seed laser only for the first pass, and then we recirculate the photon pulse from the modulator within a cavity, matching, for instance, the FLASH repetition rate of $1 \mathrm{MHz}$. This field is then used to modulate the energy of the next bunch. The other difference is that for the multipass case, we need not only energy modulation along the modulator but also power amplification that compensates for the resonator losses. This means that a longer modulator than conventionally used is required.

For this reason, in all cases, we have two modulator segments with a total length of $5.4 \mathrm{~m}$. This length is determined by the shortest wavelength, where the cavity losses are highest and at the same time the gain is smallest.

For this multi-pass scheme, an external seed laser at $10 \mathrm{~Hz}$ would be sufficient. Assuming using this repetition rate, the wavelength ranges under study were $200 \mathrm{~nm}$ to $300 \mathrm{~nm}$ and $50 \mathrm{~nm}$ to $100 \mathrm{~nm}$, where the latter could be achieved at sufficient pulse energy with a high-harmonic generation (HHG) source at $10 \mathrm{~Hz}$ [27]. At these working points, there are no gaps in delivered wavelength range, assuming that harmonics from 5th to 12th are possible. In order to reach the $4 \mathrm{~nm}$ wavelength, one should go to the 15 th harmonic. By this, the number of harmonics that need to be reached can be reduced by a factor of two.

Since initial studies $[28,29]$ supported the feasibility of this study, a more detailed study has been performed and is presented below.

All simulations are performed with GENESIS1.3, version 4 [30] while the part of the resonator is treated with OCELOT [31]. For the simulations shown here, the model includes the following steps that are implemented with OCELOT: the radiation field is propagated along drifts, its amplitude is reduced to simulate the reflectivity, it is focused and in addition, shifted longitudinally to compensate for the slippage. All these steps are repeated after every single pass in the modulator. Finally, a different random seed for shot noise calculation is set for each pass to include the effect of the bunch to bunch fluctuations as well.

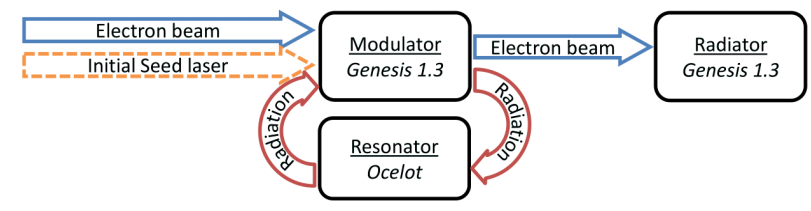

FIG. 7. Flow chart of the simulation. In the first step, an electron beam and seed pulse are used as an input for the modulator simulation. The resulting electron beam is used as an input for the amplifier simulation, while the radiation pulse is manipulated in terms of power and focusing, and used as an input for the next modulator simulation, together with a fresh electron beam.

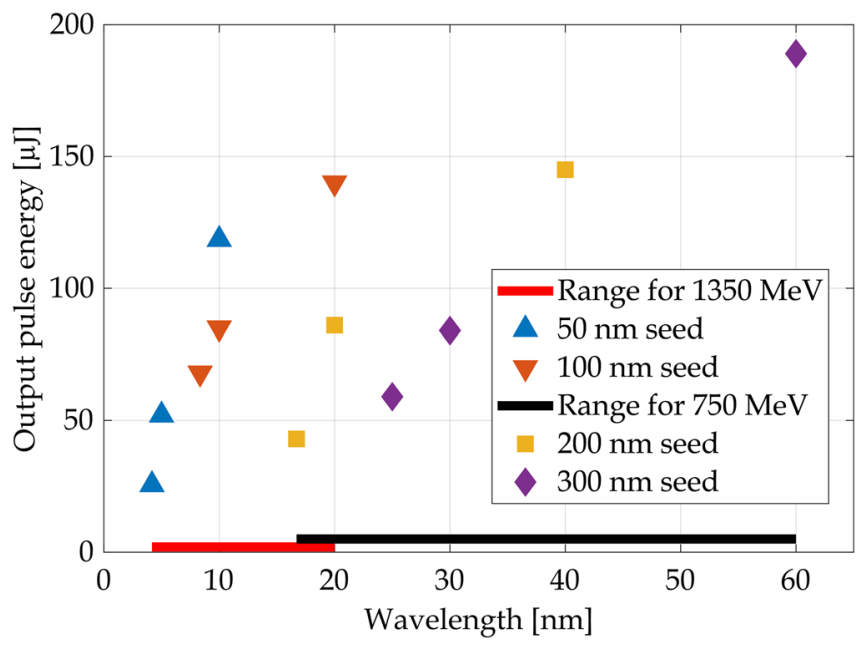

FIG. 8. Overview of output pulse energies of the 5th, 10th, and 12th harmonic for a $50 \mathrm{~nm}, 100 \mathrm{~nm}, 200 \mathrm{~nm}$, and $300 \mathrm{~nm}$ seed laser. The black bar shows the wavelength range foreseen for an electron beam energy of $750 \mathrm{MeV}$, the red bar shows the range for electron beam energies of $1350 \mathrm{MeV}$.

In all cases we assume an ideal electron beam with a longitudinally flat phase-space profile. The seed laser used for the first pass in the oscillator is an ideal Gaussian beam. An overview of the most important simulation parameters is shown in Table I. The choice of parameters is influenced by those foreseen for the future FLASH upgrade [32]. A flow chart describing the different steps of the simulations is shown in Fig. 7.

As an overview, in Fig. 8 we present the results obtained with different input seed lasers and for different harmonics in a multi-pass HGHG. For each seed laser wavelength, the 5th, 10th, and 12th harmonic are shown.

In the simulations, we achieve a stable operation of the oscillator when the total fraction of the radiation power coupled back into the modulator between $3.06 \%$ and 26.8\%, as shown in Table II.

In the following, one can find a more detailed presentation of two selected wavelengths. For each of them, we compare a SASE, a single-pass HGHG, and a multipass HGHG simulation in terms of final FEL pulse in time and frequency domain. Therefore, the pulse duration, bandwidth, peak power, and energy are compared. For all cases, we assume the same lattice and the same initial electron beam parameters. For the SASE simulation though, we show the results at saturation, therefore assuming a longer

TABLE II. Total reflectivities for different seed laser wavelengths.

\begin{tabular}{lc}
\hline \hline Seed laser wavelength & Reflectivity \\
\hline $300 \mathrm{~nm}$ & $3.1 \%$ \\
$200 \mathrm{~nm}$ & $2.8 \%$ \\
$100 \mathrm{~nm}$ & $21.1 \%$ \\
$50 \mathrm{~nm}$ & $26.8 \%$ \\
\hline \hline
\end{tabular}




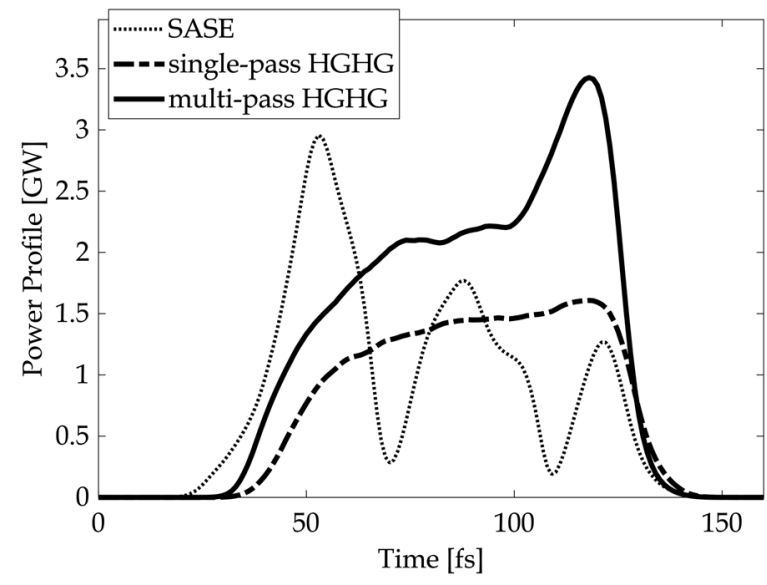

FIG. 9. Final output power profile for the three cases with the same input electron beam parameters. For the HGHG simulations, this is the 5th harmonic of $300 \mathrm{~nm}$ seed laser. For the multipass HGHG, we show the results for the 65th pass.

amplifier. The purpose is to compare the final optimized properties of SASE radiation, with the optimized seeded final radiation for the same set of initial parameters. For the single-pass HGHG, the seed laser has the same pulse duration, but its power is adjusted accordingly to achieve an energy modulation, and therefore a bunching, equal to the one achieved on average in the stabilized region of the resonator. Therefore, the setup is done in a way that a direct comparison of the two cases is possible.

\section{A. Results at $300 \mathrm{~nm}$ modulator and $60 \mathrm{~nm}$ amplifier. (5th harmonic)}

In Figs. 9 and 10 we present the results of the 5th harmonic of a $300 \mathrm{~nm}$ seed laser, therefore with an output

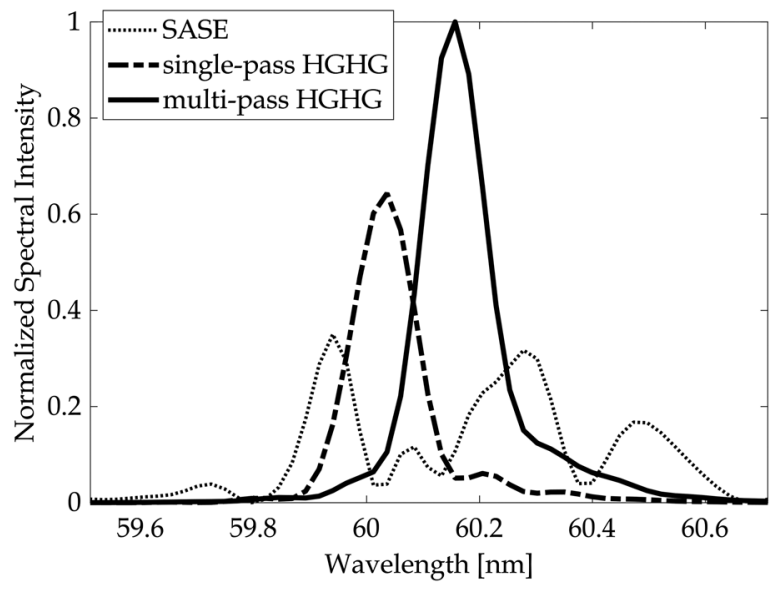

FIG. 10. Output $60 \mathrm{~nm}$ spectra for the three cases with the same lattice and input electron beam parameters. For the HGHG simulations, this is the 5th harmonic of $300 \mathrm{~nm}$ seed laser. For the multipass HGHG, we show the results for the 65 th pass. The domain shows a wavelength range of $\pm 3 \rho$ around the central wavelength of the single-pass HGHG. For the SASE simulation, the amplifier is extended to reach saturation.

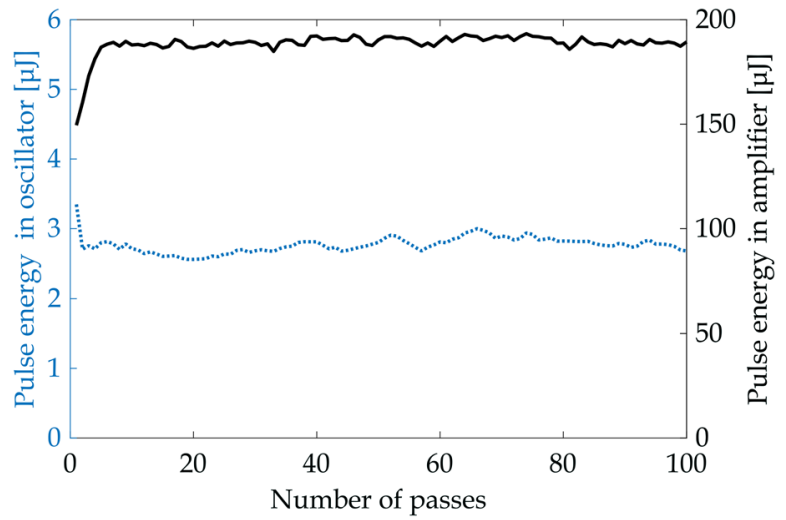

FIG. 11. Overview of the photon pulse energy per pass in the resonator (at the end of the modulator, left scale) and in the amplifier (at optimized final length, right scale). The output wavelength of the simulations is $60 \mathrm{~nm}$, which is the 5 th harmonic of $300 \mathrm{~nm}$ seed that initiates the process during the first pass only. Here we show the energy for 100 passes.

wavelength of $60 \mathrm{~nm}$. The system is stabilized after around 5 passes (see Fig. 11) with the estimated root-mean-square (RMS) fluctuations of power in the oscillator being $4.3 \%$ and in the amplifier $2.4 \%$. The key parameters for the comparison between the resulting FEL pulse from singlepass and multipass HGHG are shown in Table III. For the multipass HGHG, the 65th pass is used as an example to compare the resulting radiation in time and frequency domain and to provide the calculations shown in Table III. For a comparison, the time-bandwidth product for an ideal Gaussian beam is 0.44 . In addition, we see that the spectrum is shifting toward longer wavelengths in the multi-pass set-up (Fig. 12) and that the power is amplified more toward the head of the electron beam (Fig. 9) as a result of slippage effects. The wavelength shift is the result of an induced time dependence of the phase of the radiation pulse. Finally, the SASE output radiation has reduced longitudinal coherence and spectral intensity compared to the HGHG final seeded radiation.

There is a frequency chirp that is developed with the number of passes. As a result, the pulse duration is reduced and the peak power is increased. Depending on the wavelength, there are methods available to compensate for this effect and stretch the pulse in the time domain, for instance using gratings. However, if the pulse shortening can be

TABLE III. Overview of $60 \mathrm{~nm}$ output radiation simulations. For the multipass HGHG the pulse energy and FWHM pulse duration are the average ones in the stable regime of the oscillator. The time-bandwidth product is calculated at the 65th pass.

\begin{tabular}{lcc}
\hline \hline & Multi-pass & Single-pass \\
\hline Output pulse energy & $189 \mu \mathrm{J}$ & $115 \mu \mathrm{J}$ \\
FWHM pulse duration & $82 \mathrm{fs}$ & $78 \mathrm{fs}$ \\
Time-bandwidth product & 0.66 & 0.62 \\
\hline \hline
\end{tabular}




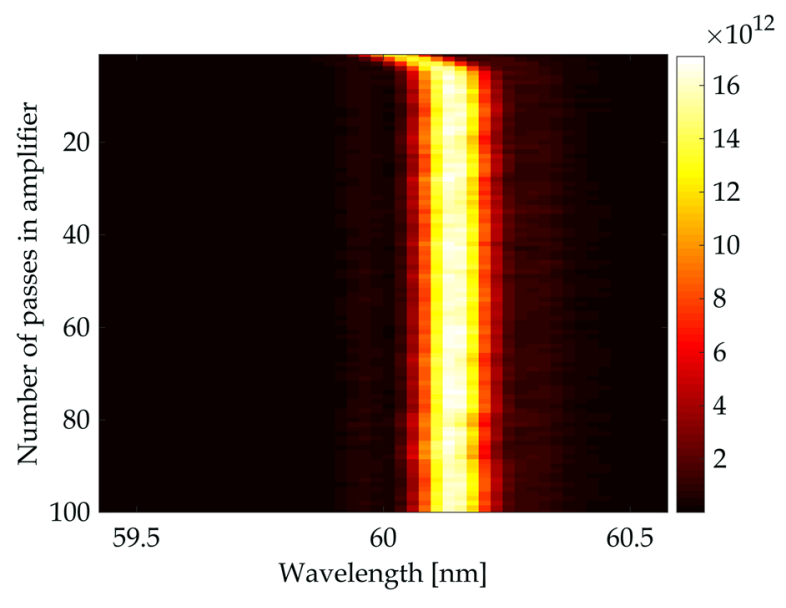

FIG. 12. All spectra for the $60 \mathrm{~nm}$ output radiation simulations. One can see that during the startup process, the spectral distribution shifts to longer wavelengths, where it stabilizes after about 5 roundtrips. The color represents the radiation intensity in arbitrary units. As in Fig. 10, a wavelength range of $\pm 3 \rho$ around the central wavelength of the single-pass HGHG is shown.

tolerated by the experiments, one can keep a simple resonator with shorter FEL pulses, in fact it might even be desirable. At the same time, there is a linear dependence of the phase in the time domain that is induced with the number of passes in the oscillator. This leads to a wavelength shift and an increased output energy, as already observed with an electron beam energy chirp in HGHG schemes before [33].

\section{B. Results at $50 \mathrm{~nm}$ modulator and $4.17 \mathrm{~nm}$ amplifier (12th harmonic)}

In Fig. 13 and 14 we show the results of a SASE, a multipass and a single-pass simulation of an output wavelength of $4.17 \mathrm{~nm}$, which is the 12th harmonic of a $50 \mathrm{~nm}$ seed

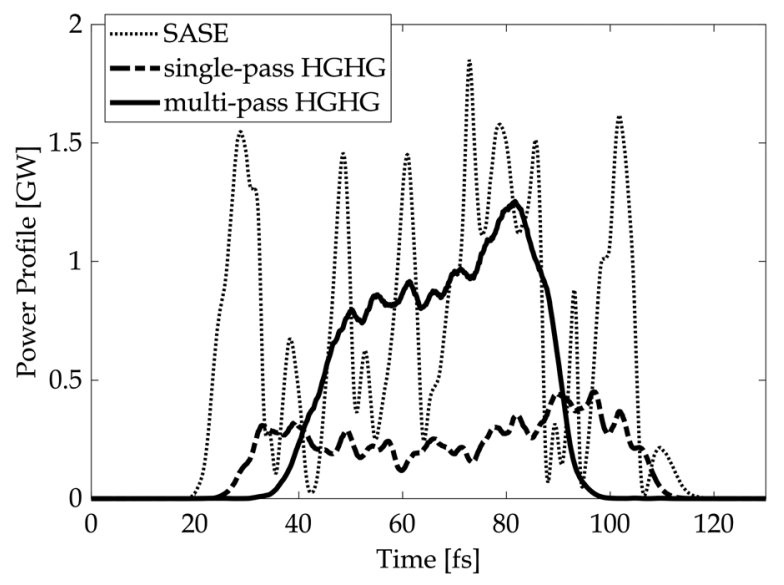

FIG. 13. Output power profile for the three cases with the same electron beam parameters. For the HGHG simulations, this is the 12th harmonic of $50 \mathrm{~nm}$ seed laser. For the multipass HGHG, we show the results for the 65 th pass.

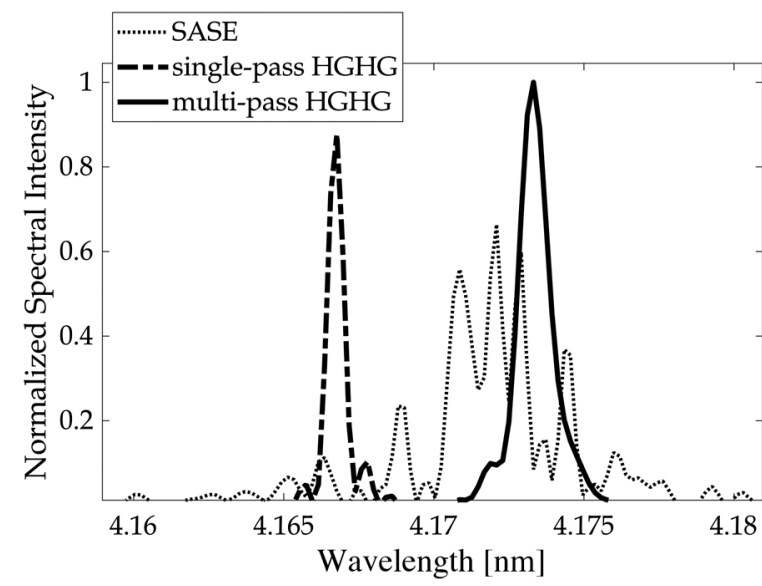

FIG. 14. Output $4.17 \mathrm{~nm}$ spectra for the three cases with the same lattice and input electron beam parameters. For the HGHG simulations, this is the 12th harmonic of $100 \mathrm{~nm}$ seed laser. For the multipass HGHG, we show the results for the 65 th pass. The domain shows a wavelength range of $\pm 3 \rho$ around the central wavelength of the single-pass HGHG. For the SASE simulation, the amplifier is extended to reach saturation.

laser. Similarly to the previous case, we show an overview of these results in Table IV. The power profile, the spectrum and the calculations of the multi-pass HGHG are based on the 65th pass, which serves as an example. For this wavelength, the clear power growth stops after roughly 35 passes (See Fig. 15). In the stable region, the RMS fluctuations of power in the oscillator are $4 \%$, while in the amplifier they are $3.2 \%$. In this case we observe a pulse shortening and a wavelength shift of the multipass HGHG, compared to the single-pass HGHG as already mentioned in the $30 \mathrm{~nm}$ case. The shifted wavelength eventually stabilizes (Fig. 16), while the number of roundtrips needed for stabilization is strongly dependent on the initial parameters which were not under further investigation in this paper.

It should be noted that the single-pass HGHG starts with a Gaussian seed laser of certain waist and Rayleigh length, while after several passes in the multi-pass HGHG, these properties of the light have changed. Therefore, it is not expected for the single-pass HGHG and the multipass HGHG to lead to the exact same results. Instead, in this paper we focus on the longitudinal coherence that is

TABLE IV. Overview of $4.17 \mathrm{~nm}$ simulations. For the multipass HGHG the pulse energy and FWHM pulse duration are the average ones in the stable regime of the oscillator. The timebandwidth product is calculated at the 65 th pass.

\begin{tabular}{lcc}
\hline \hline & Multipass & Single-pass \\
\hline Output pulse energy & $43 \mu \mathrm{J}$ & $21 \mu \mathrm{J}$ \\
FWHM pulse duration & $43 \mathrm{fs}$ & $72 \mathrm{fs}$ \\
Time-bandwidth product & 0.61 & 0.51 \\
\hline \hline
\end{tabular}




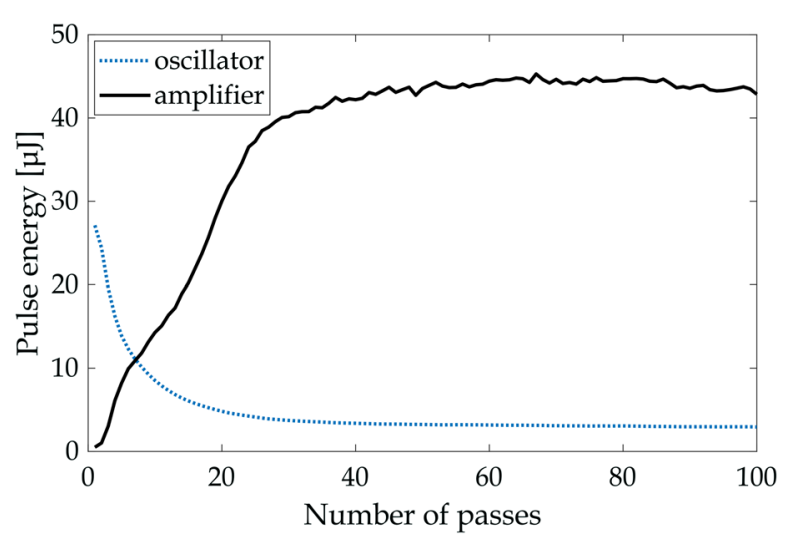

FIG. 15. Overview of the photon pulse energy per pass in the resonator (at the end of the modulator) and in the amplifier (at optimized final length). The output wavelength of the simulations is $4.17 \mathrm{~nm}$, which is the 12th harmonic of $300 \mathrm{~nm}$ seed that initiates the process during the first pass only. Here we show the energy for 100 passes.

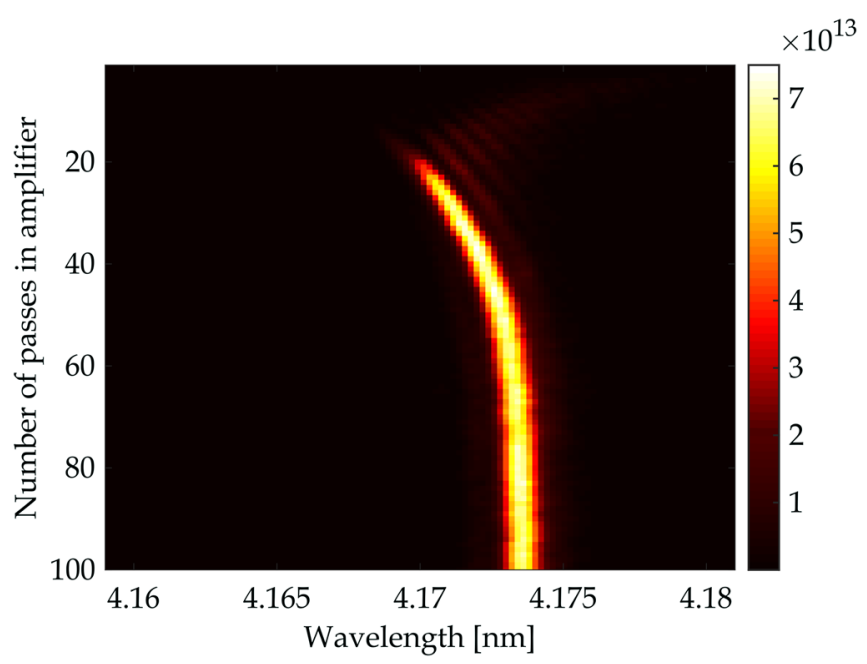

FIG. 16. All spectra for the $4.17 \mathrm{~nm}$ output radiation simulations. One can see that during the start-up process, the spectral distribution shifts to longer wavelengths, where it eventually stabilizes. Due to the initial conditions, the system needs more roundtrips to stabilize. The color represents the radiation intensity in arbitrary units. As in Fig. 14, a wavelength range of $\pm 3 \rho$ around the central wavelength of the single-pass HGHG is shown.

achieved with the multipass HGHG. As expected, the longitudinal coherence and spectral intensity of HGHG is improved compared to the SASE simulation.

\section{DISCUSSION AND OUTLOOK}

The simulations performed in IV support the assumption that an optical feedback based FEL can work in principle, and state-of-the-art optical components to build such optical feedback system do already exist. However, there are still some issues left that will be addressed in detail in future studies.

First, there is no concrete SRS design yet, including the needed in-coupling and out-coupling chicane geometry, which means simulations on designated designs have to be done. This includes damage threshold simulation, studies on the expected stability in wavelength, pointing, and length of the SRS. The latter will introduce a shot-to-shot shifting in the longitudinal position where the electron beam is hit. Assuming a region of $300 \mathrm{fs}$ in the bunch is suited for lasing, the SRS-length must not drift or jitter by more than about $90 \mu \mathrm{m}$ over the about $150 \mathrm{~m}$ long SRS at repetition rates of $1 \mathrm{MHz}$. Some classes of photon science experiments, such as time-of-flight experiments, require lower repetition rates. Methods to provide these need to be studied.

Second, the SRS HGHG scheme requires to get to an equilibrium between energy loss in the SRS and the generation of radiation in the modulator. There are several reasons that could prevent the system to maintain or reach this state: If one bunch inside a train is missing, the equilibrium is not maintained and without countermeasures, all further bunches are lost for the system.

For stable operation, ways to characterize the recirculating radiation indestructibly, e.g., using mirror leakage, need to be found as well as methods to couple the seed into the SRS. Studies on the stability, tuning range, and parameter range of the electron beam, seed laser, and SRS-components need to be performed once promising designs are found.

Finally, the application of two SRS to exploit EEHG seeding has to be investigated. Due to the nature of EEHG to enable higher harmonics, this method seems to be better suited for high-energy linacs like the EuXFEL, where such scheme is currently under study.

\section{A. Summary}

In this paper, we propose a novel method for the generation of fully coherent, stable radiation from freeelectron lasers at high repetition rates. By enclosing the modulators used for different seeding schemes with an optical cavity, the usually unused radiation pulse is fed back into the undulator were it effects the next bunch to come. We discussed the different seeding methods which could exploit our scheme and presented first simulation results for the high-gain harmonic generation scheme using a resonator for a variety of wavelengths lying in the window we want to cover in the future at FLASH [32].

\section{ACKNOWLEDGMENTS}

G. P. is paid by the Helmholtz Association of German Research Centres in the scope of the project InternLabs0002: CAS-Helmholtz International Laboratory on FreeElectron Laser Science and Technology (CHILFEL) between Deutsches Elektronen-Synchrotron (DESY), 
European XFEL and SINAP/ShanghaiTechnology University. This research was supported in part through the European XFEL and DESY funded Maxwell computational resources operated at Deutsches ElektronenSynchrotron (DESY), Hamburg, Germany.

[1] J. Rossbach, J. R. Schneider, and W. Wurth, 10 years of pioneering X-ray science at the free-electron laser flash at DESY, Phys. Rep. 808, 1 (2019).

[2] W. Ackermann et al., Operation of a free-electron laser from the extreme ultraviolet to the water window, Nat. Photonics 1, 336 (2007).

[3] H. Weise and W. Decking, Commissioning and first lasing of the European XFEL, in Proc. of International Free Electron Laser Conference (FEL'17), Santa Fe, NM, USA, August 20-25, 2017, number 38 in International Free Electron Laser Conference (JACoW, Geneva, Switzerland, 2018), pp. 9-13, https://doi.org/10.18429/JACoWFEL2017-MOC03.

[4] A. Brachmann, M. Dunham, and J. F. Schmerg, Lcls-iistatus and upgrades. In Proc. of International Free Electron Laser Conference (FEL'19), Hamburg, Germany, August 26-30, 2019, number 39 in International Free Electron Laser Conference (JACoW, Geneva, Switzerland, 2019), https://doi.org/10.18429/JACoW-FEL2019-FRA02.

[5] Z. Zhao, D. Wang, Z.-H. Yang, and L. Yin, SCLF: An 8-GeV CW SCRF Linac-Based X-Ray FEL Facility in Shanghai, in Proceedings, 38th International Free Electron Laser Conference, FEL2017, MOP055, 2018. https:// doi.org/10.18429/JACoW-FEL2017-MOP055.

[6] J. Gallardo, Proceedings of the workshop prospects for a 1 angstrom free-electron laser 1990, https://www .researchgate.net/publication/236362786_Proceedings_ of_the_workshop_prospects_for_a_1_angstrom_freeelectron_laser.

[7] G. Dattoli, B. Faatz, L. Giannessi, and P. L. Ottaviani, The tandem FEL dynamic behavior, IEEE J. Quantum Electron. 31, 1584 (1995).

[8] G. Dattoli, L. Giannessi, and P. L. Ottaviani, Mopa optical klystron FELs and coherent harmonic generation, Nucl. Instrum. Methods Phys. Res., Sect. A 507, 26 (2003).

[9] G. Dattoli, L. Giannessi, and P. Ottaviani, Oscillatoramplifier free electron laser devices with stable output power, J. Appl. Phys. 95, 3211 (2004).

[10] H. P. Freund, D. C. Nguyen, P. Sprangle, and P. J. M. van der Slot, Three-dimensional, time-dependent simulation of a regenerative amplifier free-electron laser, Phys. Rev. Accel. Beams 16, 010707 (2013).

[11] M. W. Reinsch, G. Penn, P. Gandhi, and J. Wurtele, The radiator-first HGHG multi-mhz X-ray FEL concept, Proc. of Free-Electron Laser Conference (FEL'12), Nara, Japan, August 26-31, 2012, Number 34 in International Free Electron Laser Conference, TUPD19, pages 273276, 2012, http://accelconf.web.cern.ch/FEL2012/html/ sessi0n.htm.

[12] L. H. Yu, M. Babzien, I. Ben-Zvi, L. Dimauro, A. Doyuran, W. Graves, E. Johnson, S. Krinsky, R. Malone, I. Pogorelsky, J. Skaritka, G. Rakowsky, L. Solomon, X. J. Wang, M.
Woodle, V. Yakimenko, S. Biedron, J. N. Galayda, E. Gluskin, and I. Vasserman, High-gain harmonic-generation free-electron laser, Science 289, 932 (2000).

[13] L. H. Yu and J. Wu, Theory of high gain harmonic generation: An analytical estimate, Nucl. Instrum. Methods Phys. Res., Sect. A 483, 493 (2002).

[14] E. Allaria, P. Cinquegrana, S. Cleva, D. Cocco, M. Cornacchia, P. Craievich, I. Cudin, G. D'Auria, M. Dal Forno, M. Danailov, R.e De Monte, G. De Ninno, P. Delgiusto, A. Demidovich, S. Di Mitri, B. Diviacco, A. Fabris, R. Fabris, W. Fawley, and M. Zangrando, Highly coherent and stable pulses from the Fermi seeded freeelectron laser in the extreme ultraviolet, Nat. Photonics 6 , 699 (2012).

[15] K. Hacker et al., First Lasing of an HGHG seeded FEL at FLASH, in Proceedings, 37th International Free Electron Laser Conference (FEL 2015): Daejeon, Korea, August 23-28, 2015, p. WEP030, 2015. https://doi.org/10.18429/ JACoW-FEL2015-WEP030.

[16] J. Boedewadt, C. Lechner, R. Assmann, A. Azima, M. Drescher, N. Ekanayake, B. Faatz, K. Hacker, M. Kazemi, I. Hartl, S. Khan, T. Laarmann, T. Maltezopoulos, T. Plath, and J. Rossbach, Operation of a seeded XUV free electron laser at DESY with high-gain harmonic generation seeding. OSA technical digest (online), page JTu4L.5 (2017); CLEO: Applications and Technology, (San Jose California), 14 May 2017-19 May 2017, OSA. ISBN 978-1-94358027-9, https://doi.org/10.1364/CLEO_AT.2017.JTu4L.5.

[17] S. Reiche, Overview of seeding methods for FELs, in Proceedings of the 4th International Particle Accelerator Conference, IPAC-2013, Shanghai, China, 2013 (JACoW, Shanghai, China, 2013), pp. 2063-2067.

[18] J. Wu and L.-H. Yu, Coherent hard x-ray production by cascading stages of high gain harmonic generation, Nucl. Instrum. Methods Phys. Res., Sect. A 475, 104 (2001).

[19] B. Liu et al., Demonstration of a widely-tunable and fullycoherent high-gain harmonic-generation free-electron laser, Phys. Rev. Accel. Beams 16, 020704 (2013).

[20] E. Allaria, D. Castronovo, P. Cinquegrana, P. Craievich, M. Dal Forno, M. Danailov, G. D'Auria, A. Demidovich, G. De Ninno, S. Di Mitri, B. Diviacco, W. Fawley, M. Ferianis, E. Ferrari, L. Froehlich, G. Gaio, D. Gauthier, L. Giannessi, R. Ivanov, and M. Zangrando, Two-stage seeded soft-X-ray free-electron laser, Nat. Photonics 7, 913 (2013).

[21] D. Xiang and G. Stupakov, Echo-enabled harmonic generation free electron laser, Phys. Rev. Accel. Beams 12, 030702 (2009).

[22] C. Feng et al., Coherent extreme ultraviolet free-electron laser with echo-enabled harmonic generation, Phys. Rev. Accel. Beams 22, 050703 (2019).

[23] P. Ribič, A. Abrami, L. Badano, M. Bossi, H.-H. Braun, N. Bruchon, F. Capotondi, D. Castronovo, M. Cautero, P. Cinquegrana, M. Coreno, M. Couprie, I. Cudin, M. Danailov, G. De Ninno, A. Demidovich, S. Di Mitri, B. Diviacco, W. Fawley, and E. Allaria, Coherent soft x-ray pulses from an echo-enabled harmonic generation freeelectron laser, Nat. Photonics 13, 1 (2019). 
[24] B. Faatz, E. Plönjes, S. Ackermann, A. Agababyan, V. Asgekar, V. Ayvazyan, S. Baark, N. Baboi, V. Balandin, N. Bargen, Y. Bican, O. Bilani, J. Bödewadt, M. Böhnert, R. Böspflug, S. Bonfigt, H. Bolz, F. Borges, O. Borkenhagen, and J. Zemella, Simultaneous operation of two soft x-ray free-electron lasers driven by one linear accelerator, New J. Phys. 18, 062002 (2016).

[25] T. Plath, P. Amstutz, J. Bödewadt, G. Brenner, N. Ekanayake, B. Faatz, K. Hacker, K. Honkavaara, L. Lazzarino, C. Lechner, T. Maltezopoulos, M. Scholz, S. Schreiber, M. Vogt, J. Zemella, and T. Laarmann, Freeelectron laser multiplex driven by a superconducting linear accelerator, J. Synchrotron Radiat. 23, 1070 (2016).

[26] B. Faatz, M. Braune, O. Hensler, K. Honkavaara, R. Kammering, M. Kuhlmann, E. Ploenjes, J. Roensch-Schulenburg, E. Schneidmiller, S. Schreiber, K. Tiedtke, M. Tischer, R. Treusch, M. Vogt, W. Wurth, J. Zemella, and M. Yurkov, The FLASH Facility: Advanced options for FLASH2 and future perspectives, Appl. Sci. 7, 1114 (2017).

[27] E. Takahashi, Y. Nabekawa, and K. Midorikawa, Generation of $10-\mu \mathrm{j}$ coherent extreme-ultraviolet light by use of high-order harmonics, Opt. Lett. 27, 1920 (2002).

[28] G. Paraskaki, S. Ackermann, B. Faatz, V. Grattoni, C. Lechner, M. Mehrjoo, G. Geloni, S. Serkez, T. Tanikawa, and W. Hillert, Study of a seeded oscillator-amplifier FEL, in Proc. of International Free Electron Laser Conference (FEL'19), Hamburg, Germany, August 26-30, 2019, number 39 in International Free Electron Laser Conference (JACoW, Geneva, Switzerland, 2019).

[29] S. Ackermann, B. Faatz, V. Grattoni, C. Lechner, G. Paraskaki, G. Geloni, S. Serkez, T. Tanikawa, and W. Hillert, High-repetition-rate seeding schemes using a resonator-amplifier setup, in Proc. of International Free Electron Laser Conference (FEL'19), Hamburg, Germany, August 26-30, 2019, number 39 in International Free Electron Laser Conference (JACoW, Geneva, Switzerland, 2019).

[30] S. Reiche, Genesis 1.3: A fully 3d time-dependent FEL simulation code, Nucl. Instrum. Methods Phys. Res., Sect. A 429, 243 (1999).

[31] I. Agapov, G. Geloni, S. Tomin, and I. Zagorodnov, OCELOT: A software framework for synchrotron light source and FEL studies, Nucl. Instrum. Methods Phys. Res., Sect. A 768, 151 (2014).

[32] M. Beye, FLASH2020+: Making FLASH brighter, faster and more flexible: Conceptual Design Report, Verlag Deutsches Elektronen-Synchrotron, Hamburg, 2020. ISBN 9783945931301. https://doi.org/10.3204/PUBDB2020-00465.

[33] G. Paraskaki, S. Ackermann, B. Faatz, V. Grattoni, W. Hillert, C. Lechner, and J. Zemella, Impact of electron beam energy chirp on seeded FELs in 39th International Free-Electron Laser Conference, Hamburg (Germany), 26 Aug 2019-30 Aug 2019 (JACoW Publishing, Geneva, Switzerland, 2019), pp. 238-241. 